\title{
Clinical features and relapse risks of lgG4- related ophthalmic disease: a single-center experience in China
}

Zhen Zhao ${ }^{1+}$, Dapeng Mou ${ }^{2+}$, Ziqiao Wang ${ }^{1}$, Qiaozhu Zeng ${ }^{1}$, Zhenfan Wang ${ }^{1}$, Jimeng Xue ${ }^{1}$, Limin Ren ${ }^{1}$, Yanying Liu $^{1 *}$ and Yin Su${ }^{1 *}$

\begin{abstract}
Background: IgG4-related ophthalmic disease (IgG4-ROD) is one of the phenotypes of IgG4-related disease (IgG4$\mathrm{RD}$ ), and its lesions are mainly located in the ocular. Currently, there are few studies on IgG4-ROD and no study has compared the phenotypic differences between lgG4-ROD and non lgG4-ROD (nlgG4-ROD). Thus, it is difficult to establish the optimal treatment strategy for lgG4-ROD. The aim of this study was to identify the disparities between the two groups and to clarify the risk factors for IgG4-ROD relapse.

Methods: 434 lgG4-RD patients met comprehensive diagnostic criteria and diagnosed at Peking University People's Hospital between January 2009 and January 2020 were recruited in this study. Patients were divided into IgG4-ROD and nlgG4-ROD group according to the ophthalmic involvement. Demographic, clinical, and laboratory data of two groups were collected and compared. Cox regression analysis was used to identify the independent risk factors for IgG4-ROD relapse.

Results: 255 lgG4-ROD patients were identified in this study. IgG4-ROD group had almost equal sex ratio, younger age of disease onset and diagnosis comparing with nlgG4-ROD patients. As compared to nlgG4-ROD group, higher percentage of IgG4-ROD patients met the 2019 American College of Rheumatology/European League Against Rheumatism classification criteria (AECC) for lgG4-RD; moreover, lgG4-ROD patients had higher AECC scores and IgG4-RD responder index (RI). Allergic diseases and multiorgan involvement were more common in IgG4-ROD group. IgG4-ROD was frequently associated with salivary gland, paranasal sinus, lung, and lymph node involvement, while retroperitoneal fibrosis and biliary system lesions were more common in nlgG4-ROD. IgG4-ROD patients had higher serum lgG4 levels, IgG4/lgG ratio, IgE levels, and lower CRP levels. The initial glucocorticoid plus immunosuppressant was a protective factor for IgG4-ROD relapse. IgG4-ROD patients treated with initial glucocorticoid plus immunosuppressant had longer relapse-free survival time than patients treated with initial glucocorticoid monotherapy.

(Continued on next page)
\end{abstract}

\footnotetext{
*Correspondence: liuyanying20030801@msn.com; suyin0921@163.com

†Zhen Zhao and Dapeng Mou contributed equally to this work.

'Department of Rheumatology and Immunology, Peking University People's

Hospital, 11 Xizhimen South Street, Beijing 100044, China

Full list of author information is available at the end of the article
}

\section{$\triangle B M C$}

(c) The Author(s). 2021 Open Access This article is licensed under a Creative Commons Attribution 4.0 International License, which permits use, sharing, adaptation, distribution and reproduction in any medium or format, as long as you give appropriate credit to the original author(s) and the source, provide a link to the Creative Commons licence, and indicate if changes were made. The images or other third party material in this article are included in the article's Creative Commons licence, unless indicated otherwise in a credit line to the material. If material is not included in the article's Creative Commons licence and your intended use is not permitted by statutory regulation or exceeds the permitted use, you will need to obtain permission directly from the copyright holder. To view a copy of this licence, visit http://creativecommons.org/licenses/by/4.0/ The Creative Commons Public Domain Dedication waiver (http://creativecommons.org/publicdomain/zero/1.0/) applies to the data made available in this article, unless otherwise stated in a credit line to the data. 
(Continued from previous page)

Conclusions: IgG4-ROD patients had distinctive clinical features compared with nlgG4-ROD patients. The initial glucocorticoid plus immunosuppressant was a protective factor for lgG4-ROD relapse, which could prolong the relapse-free survival time of IgG4-ROD patients. These findings may have important implications for understanding and management of IgG4-ROD.

Keywords: IgG4-related ophthalmic disease, Clinical features, Phenotypic differences, Relapse risks

\section{Background}

IgG4-related disease (IgG4-RD) is a new clinicopathological entity, originally proposed by Kamisawa et al. in 2003 [1]. Although the exact etiology and pathogenesis remain largely unknown, remarkable progress has been made in the understanding of this disease. Now it has been recognized as an immune-mediated systemic fibroinflammatory disease featuring tumefactive lesions [2]. Serum IgG4 level is elevated in the majority of patients with IgG4-RD, but this is a nonspecific finding [3]. Characteristic histopathologic features are lymphoplasmacytic infiltration rich in IgG4-immunopositive plasma cells, storiform fibrosis, and obliterative phlebitis $[4,5]$. IgG4-RD is a highly heterogenous disorder that can affect almost every organ system in the body [6-9], and the ocular lesions are also extremely common.

In the past, different terms have been used to name IgG4-RD with ocular lesions [10-14]. However, with the publication of comprehensive diagnostic criteria for IgG4-RD in 2012 [15], IgG4-related ophthalmic disease (IgG4-ROD) has been regarded as a standardized name for IgG4-RD with ophthalmic involvement. The ocular anatomic structures affected in IgG4-ROD include lacrimal gland, soft tissue, extraocular muscle, eyelid, optical nerve, orbital bone, sclera, conjunctiva, and trigeminal nerve, and extraocular involvement can also occur with ocular lesions simultaneously or metachronously [16]. We define these cases who fulfill comprehensive diagnostic criteria for IgG4-RD but have no ocular lesions as non IgG4-ROD (nIgG4-ROD). So IgG4-RD can be divided into IgG4-ROD and nIgG4-ROD according to whether there is ophthalmic involvement. Currently, there has been no study to explore the differences in clinical features between IgG4-ROD and nIgG4-ROD patients.

The use of systemic glucocorticoids as first-line therapy for IgG4-ROD usually leads to an effective initial response [17]. However, the relapse rate of IgG4-ROD is quite high during glucocorticoid tapering or withdrawal [18]. Therefore, prolonged the use of glucocorticoids is necessary to prevent relapse, but it often leads to many steroid-related adverse events. Immunosuppressants or biological agent rituximab as a second or third-line treatment has been effectively used in the treatment of recurrent or refractory IgG4-ROD patients [17, 18].
However, the optimal treatment strategy for IgG4-ROD has not yet been established.

In this study, we focused on comparing the clinical features between IgG4-ROD and nIgG4-ROD patients and exploring the risk factors of IgG4-ROD patients. The aim was to identify the disparities between the two groups and to clarify the risk factors for IgG4-ROD relapse, so as to further improve the understanding of IgG4-RD with different phenotypes and help to better formulate diagnosis and management strategies.

\section{Methods \\ Study population}

This retrospective study was conducted in the Peking University People's Hospital, a total of 434 patients with IgG4-RD who have attended at our hospital between January 2009 and January 2020 were recruited. Patients with infections, tumors, or other autoimmune diseases were excluded from this study. All enrolled patients met the IgG4-RD comprehensive diagnostic criteria [15]: (1) clinical examination shows characteristic diffuse/localized swelling or masses in single or multiple organs, (2) hematological examination shows elevated serum IgG4 concentrations (>135 mg/dl), and (3) histopathologic examination shows (a) marked lymphocyte and plasmocyte infiltration and fibrosis or (b) infiltration of IgG4+ plasma cells (ratio of IgG4+/IgG+ cells $>40 \%$ and $>10$ IgG4+ plasma cells/high power field). Whether the clinical symptoms, signs, physical examinations, or imaging tests including ultrasound, computed tomography (CT), magnetic resonance imaging (MRI) or positron emission tomography/computed tomography (PET/CT) revealed ophthalmic involvement in a patient with IgG4-RD, the patient was considered to have IgG4-ROD. Patients were divided into IgG4-ROD and nIgG4-ROD group according to the ophthalmic involvement. This study was approved by the Medical Ethics Committee of Peking University People's Hospital (Beijing, China).

\section{Data collection}

All patients' clinical data including age, sex, history of allergic disease, initial lesion site, number of organs involved, and ophthalmic symptoms were collected from medical records. IgG4-RD responder index (RI) [19] and 2019 American College of Rheumatology/European 
League Against Rheumatism classification criteria (AECC) for IgG4-RD [20] scores were calculated. The diagnosis of allergic disease was made by specialists based on the criteria of the European Academy of Allergy and Clinical Immunology. The laboratory parameters including complete blood count, liver function, serum IgG, serum IgG4, serum IgE, antinuclear antibody (ANA), rheumatoid factor (RF), erythrocyte sedimentation rate (ESR), C-reactive protein (CRP), and complement tests were analyzed retrospectively. Different imaging tests such as ultrasound, CT, MRI, or PET/CT were performed in all patients to evaluate the organ involvement. Totally, 240 patients underwent tissue biopsy, and the samples were fixed with formaldehyde solution, routine paraffin embedding, and hematoxylineosin staining. Immunohistochemical staining was performed using antibodies to IgG and IgG4. The pathological diagnosis of IgG4-RD was confirmed by the experienced pathologists after ruling out all potential alternate diagnoses. The initial medication regimens, duration of immunosuppressants, and the relapse and relapse-free survival time during follow-up period of IgG4-ROD patients were reviewed. Data regarding patients' clinical features of the two groups were compared. Cox regression analysis was used to identify the independent risk factors for IgG4-ROD relapse.

\section{Statistical analysis}

The quantitative variables were described as mean \pm standard deviation (SD) or median (interquartile range, IQR). Categorical variables were presented as proportions. Differences between groups were analyzed using two independent samples $t$ test or Wilcoxon rank sum test for quantitative variables, and chi-square test or Fisher's exact test for categorical variables. Univariate and multivariate Cox regression analyses (Enter method) were performed to identity the possible risk factors for IgG4-ROD relapse. Variables with $P<0.2$ in the univariate analysis were included in the multivariate analysis. The Kaplan-Meier survival curve and log-rank test were used to compare the difference of the initial treatment options on the relapse of IgG4-ROD. All the data were processed by the IBM SPSS Statistics version 23.0 software. Significant differences were defined as $P<0.05$.

\section{Results}

\section{Demographic characteristics}

Altogether, 434 patients with IgG4-RD were recruited in this study including 247 males and 187 females. Respectively, 212 (48.8\%), 28 (6.5\%), and 194 (44.7\%) patients were diagnosed as definite, probable, and possible IgG4$\mathrm{RD}$, according to the IgG4-RD comprehensive diagnostic criteria. Among these patients, 255 cases were identified with IgG4-ROD and another 179 cases were diagnosed with nIgG4-ROD. The demographic characteristics of the patients are displayed in Table 1 . We could observe several differences between IgG4-ROD and nIgG4-ROD patients. The sex ratio of male to female in the IgG4ROD group was approximately equal (0.96:1), lower than that in nIgG4-ROD group $(2.14: 1)(P<0.001)$. The mean age of disease onset in IgG4-ROD group and nIgG4ROD group was $51.1 \pm 13.2$ years and $55.5 \pm 13.5$ years, respectively, suggesting the former had an earlier disease onset $(P=0.001)$. The mean age of disease diagnosis in IgG4-ROD group was $54.4 \pm 12.6$ years, which was

Table 1 Demographic and clinical features of the patients

\begin{tabular}{|c|c|c|c|c|}
\hline Characteristics & IgG4-RD & IgG4-ROD & nlgG4-ROD & $P$ value \\
\hline Number of cases, $n$ & 434 & 255 & 179 & \\
\hline Male to female & $1.32: 1$ & $0.96: 1$ & $2.14: 1$ & $<0.001^{*}$ \\
\hline AECC score & $33.0 \pm 14.2$ & $37.0 \pm 12.9$ & $27.2 \pm 14.0$ & $<0.001^{*}$ \\
\hline AECC score $\geq 20, n(\%)$ & $344(79.3)$ & 228(89.4) & $116(64.8)$ & $<0.001^{*}$ \\
\hline Age of disease onset (years), mean \pm SD & $52.9 \pm 13.5$ & $51.1 \pm 13.2$ & $55.5 \pm 13.5$ & $0.001^{*}$ \\
\hline Age of disease diagnosis (years), mean $\pm \mathrm{SD}$ & $55.4 \pm 12.9$ & $54.4 \pm 12.6$ & $56.9 \pm 13.4$ & 0.054 \\
\hline History of allergic disease, $n(\%)$ & 214(49.3) & $167(65.5)$ & $47(26.3)$ & $<0.001^{*}$ \\
\hline lgG4-RD Rl & $11.0 \pm 6.2$ & $11.4 \pm 6.0$ & $10.2 \pm 6.3$ & $0.045^{*}$ \\
\hline Number of involved organs, median (IQR) & $5(3-6)$ & $6(4-7)$ & $3(2-5)$ & $<0.001^{*}$ \\
\hline \multicolumn{5}{|l|}{ Number of involved organs, $n$ (\%) } \\
\hline 1 organ involved & $30(6.9)$ & $4(1.6)$ & $26(14.5)$ & $<0.001^{*}$ \\
\hline 2 organs involved & $52(12.0)$ & $11(4.3)$ & $41(22.9)$ & $<0.001^{*}$ \\
\hline 3 organs involved & $46(10.6)$ & $20(7.8)$ & $26(14.5)$ & $0.026^{*}$ \\
\hline$\geq 4$ organs involved & $306(70.5)$ & $220(86.3)$ & $86(48.0)$ & $<0.001^{*}$ \\
\hline
\end{tabular}

The $P$ value refers to the comparison between IgG4-ROD and nlgG4-ROD patients. AECC American College of Rheumatology/European League Against Rheumatism classification criteria, IgG4-RD RI IgG4-RD responder index, SD standard deviation, IQR interquartile range. ${ }^{*} P<0.05$ 
younger than that in nIgG4-ROD group, but there was no statistical difference between the two groups (Table 1).

\section{Clinical features}

As compared to nIgG4-ROD group, higher percentage of IgG4-ROD patients met the 2019 AECC for IgG4-RD $(P<0.001)$; moreover, IgG4-ROD patients had higher AECC scores $(P<0.001)$ and IgG4-RD RI $(P=0.045)$. Notably, the prevalence of allergic diseases (including allergic rhinitis, asthma, and urticaria) in IgG4-ROD group was higher than that in nIgG4-ROD group $(P<0.001)$ (Table 1). The initial lesion sites at disease onset of two groups are shown in Fig. 1a. There was a significant difference in the constituent ratio of the initial lesion site between the two groups. It was found that the three most common initial lesion sites in IgG4-ROD patients were the ocular $(41.6 \%)$, salivary gland $(34.9 \%)$, and pancreas $(9.0 \%)$, while those in nIgG4-ROD patients were the pancreas $(32.9 \%)$, salivary gland $(23.4 \%)$, and retroperitoneal fibrosis $(16.8 \%)$.
In this study, we observed that a single organ involvement was very rare, and most patients $(93.1 \%)$ had multiple organ involvement. By comparison, it was found that the median number of involved organs and the percentage of $\geq 4$ organs involved in IgG4-ROD group were significantly higher than those in nIgG4-ROD group (Table 1). The involvement of other organs except for ocular is displayed in Fig. 1b. After comparison, we found that the percentages of patients with the salivary gland, paranasal sinus, lung, and lymph node involvement in IgG4-ROD group were higher than those in nIgG4-ROD group, while nIgG4-ROD patients had higher percentages of retroperitoneal fibrosis and biliary system lesions.

We also analyzed the ophthalmic symptoms and the affected ocular anatomic structures of IgG4-ROD patients (Fig. 2). Eyelid swelling (74.1\%) was the most frequent ophthalmic symptom, followed by xeropthalmia (49.4\%) and proptosis $(12.2 \%)$, while decrease vision $(2.7 \%)$ was relatively rare (Fig. 2a). The affected ocular anatomic structure in IgG4-ROD patients was evaluated in detail. Highly consistent with the ophthalmic symptoms, the

a
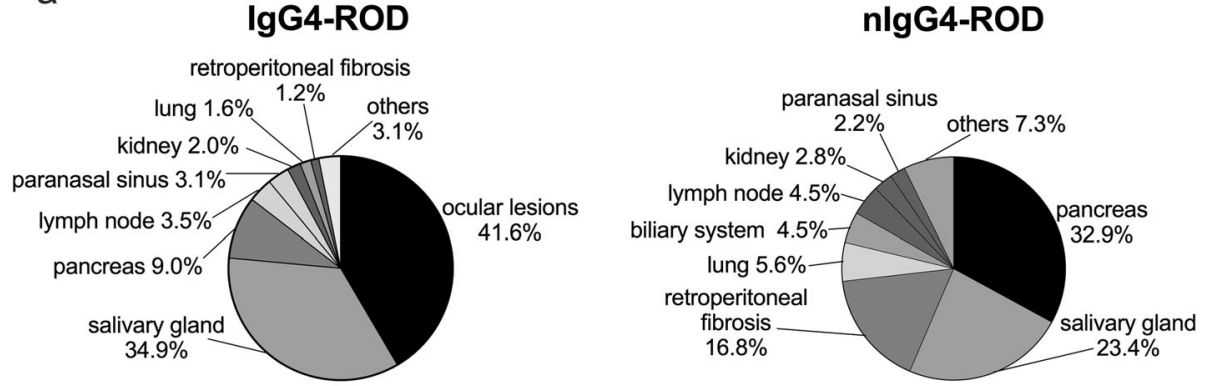

b

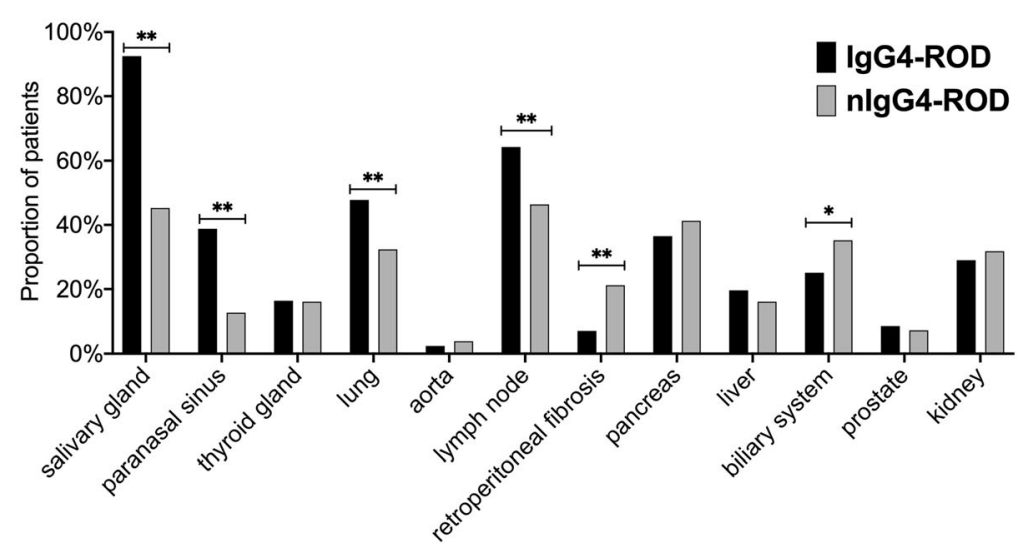

Fig. 1 The initial lesion site and organ involvement of patients with IgG4-ROD and nlgG4-ROD. a The constituent ratio of initial lesion site in IgG4-ROD and nlgG4-ROD patients. The three most frequent initial lesions in IgG4-ROD patients were the ocular (41.6\%), salivary gland (34.9\%), and pancreas (9.0\%), while in nlgG4-ROD patients were the pancreas (32.9\%), salivary gland (23.4\%), and retroperitoneal fibrosis (16.8\%). b Comparison of organ involvement except for ocular between patients with lgG4-ROD and nlgG4-ROD. The percentages of patients with the salivary gland, paranasal sinus, lung, and lymph node involvement in lgG4-ROD group were higher than those in nlgG4-ROD group, while nlgG4ROD patients had higher percentages of retroperitoneal fibrosis and biliary system lesions. ${ }^{*} P<0.05,{ }^{*} P<0.01$ 
a

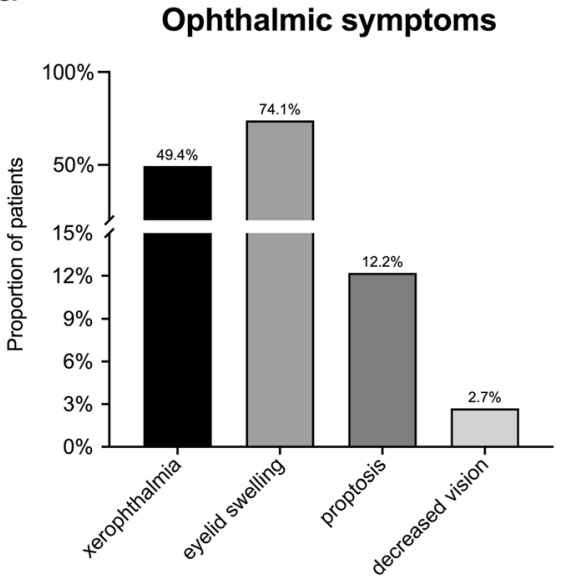

b

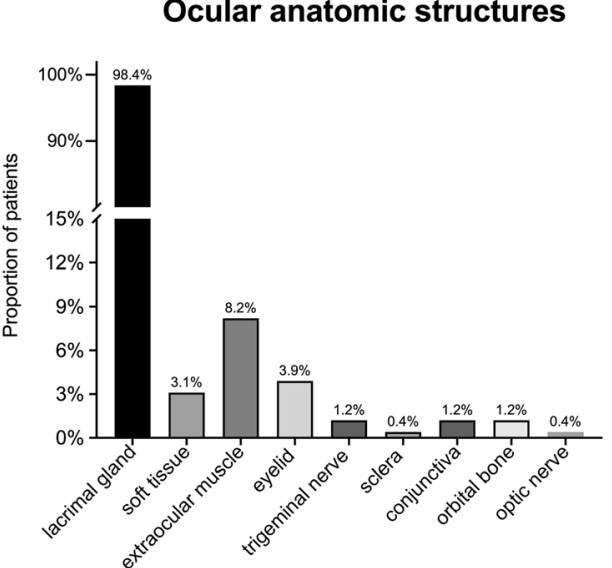

Fig. 2 Rate of ophthalmic symptoms and affected ocular anatomic structures in IgG4-ROD patients. a Proportions of patients with different ophthalmic symptoms in IgG4-ROD patients. The most frequent ophthalmic symptom was eyelid swelling (74.1\%), followed by xeropthalmia (49.4\%) and proptosis (12.2\%), while decrease vision (2.7\%) was relatively rare. b Proportions of patients with different ocular anatomic structures involved in IgG4-ROD patients. The lacrimal gland (98.4\%) occupied the most frequently affected ocular anatomic structure, followed by the extraocular muscle (8.2\%), while the eyelid (3.9\%), soft tissue (3.1\%), conjunctiva (1.2\%), trigeminal nerve (1.2\%), orbital bone (1.2\%), sclera (0.4\%), and optic nerve $(0.4 \%)$ were relatively rare.

lacrimal gland (98.4\%) occupied the most frequent affected ocular anatomic structure in IgG4-ROD patients, followed by extraocular muscle (8.2\%), while the eyelid (3.9\%), soft tissue (3.1\%), conjunctiva (1.2\%), trigeminal nerve $(1.2 \%)$, orbital bone $(1.2 \%)$, sclera $(0.4 \%)$, and optic nerve $(0.4 \%)$ were relatively rare (Fig. $2 b)$.

\section{Laboratory parameters}

The laboratory parameters of the two groups are summarized in Table 2. There were statistical differences in IgG4, IgG4/IgG ratio, IgE, and CRP between two groups. IgG4-ROD patients had higher serum IgG4 levels $(P<$ $0.001)$ and $\operatorname{IgG} 4 / \operatorname{IgG}$ ratio $(P<0.001)$ than nIgG4-ROD

Table 2 Comparison of laboratory parameters between patients with IgG4-ROD and nlgG4-ROD

\begin{tabular}{|c|c|c|c|}
\hline Laboratory parameter & IgG4-ROD & nlgG4-ROD & $P$ value \\
\hline Low serum C3, n (\%) & $87(39.7)$ & $51(34.7)$ & 0.330 \\
\hline Low serum C4, n (\%) & $75(34.7)$ & $44(29.9)$ & 0.340 \\
\hline Hypocomplementemia, n (\%) & $100(45.9)$ & $63(42.9)$ & 0.570 \\
\hline Elevated ESR, $n(\%)$ & $94(49.5)$ & 78(55.3) & 0.293 \\
\hline Elevated CRP, n (\%) & $30(16.3)$ & 45(31.9) & $0.001^{*}$ \\
\hline Elevated globulin, $n$ (\%) & $54(28.6)$ & $41(29.1)$ & 0.920 \\
\hline Eosinophilia, $n(\%)$ & $60(27.8)$ & $33(21.6)$ & 0.176 \\
\hline $\mathrm{RF}+, n(\%)$ & $43(20.5)$ & $26(18.7)$ & 0.684 \\
\hline ANA,$+ n(\%)$ & $32(14.3)$ & $25(16.7)$ & 0.542 \\
\hline Elevated IgE, n (\%) & $178(84.0)$ & $77(71.3)$ & $0.008^{*}$ \\
\hline $\mathrm{C} 3(\mathrm{~g} / \mathrm{L})$, mean $\pm \mathrm{SD}$ & $0.852 \pm 0.280$ & $0.935 \pm 0.319$ & 0.053 \\
\hline $\mathrm{C} 4(\mathrm{~g} / \mathrm{L})$, mean $\pm \mathrm{SD}$ & $0.184 \pm 0.095$ & $0.200 \pm 0.093$ & 0.115 \\
\hline ESR (mm/h), median (IQR) & $18.5(8.0-40.0)$ & 23.5(10.0-59.0) & 0.058 \\
\hline CRP (mg/L), median (IQR) & $2.24(1.00-4.70)$ & $3.05(1.26-12.45)$ & $0.004^{*}$ \\
\hline Globulin (g/L), median (IQR) & $33.7(29.7-41.5)$ & $35.4(29.8-41.5)$ & 0.808 \\
\hline Serum lgG4 (mg/dl), median (IQR) & 983(409-1838) & $523(225-1370)$ & $<0.001$ \\
\hline Serum IgG (g/L), median (IQR) & $17.5(14.3-23.2)$ & $17.2(14.1-22.0)$ & 0.609 \\
\hline lgG4/lgG, median (IQR) & $0.529(0.272-0.879)$ & $0.294(0.143-0.629)$ & $<0.001$ \\
\hline Serum IgE (IU/ml), median (IQR) & $339.7(160.8-847.6)$ & 259.4(83.1-653.3) & $0.022^{*}$ \\
\hline
\end{tabular}

$S D$ standard deviation, IQR interquartile range. ${ }^{*} P<0.05$ 
patients. IgG4-ROD group also had higher serum IgE levels and the higher proportion of patients with elevated IgE than nIgG4-ROD group $(P=0.022$ and $P=$ 0.008 , respectively). While nIgG4-ROD patients had higher CRP levels and the higher proportion of patients with elevated CRP $(P=0.004$ and $P=0.001$, respectively $)$. In addition, higher ESR levels were also found in nIgG4ROD group, but there was no statistical difference between two groups. In our study, no significant differences were found in the eosinophilia, complement, globulin, IgG, ANA, and RF results between the two groups.

\section{Treatment and relapse of IgG4-ROD}

Univariate Cox regression analysis revealed that sex, age of disease onset, and age of disease diagnosis; levels of serum C4, ESR, CRP, and IgG4; and initial treatment options were associated with IgG4-ROD relapse (Table 3). Multivariate Cox regression analysis identified initial glucocorticoid plus immunosuppressant was a protective factor for IgG4-ROD relapse (Fig. 3a). The Kaplan-Meier curves of relapse-free survival of IgG4-ROD patients based on the initial treatment options were plotted in Fig. 3b. According to the initial treatment options, IgG4-

Table 3 Univariate Cox regression analysis for lgG4-ROD relapse

\begin{tabular}{|c|c|c|c|}
\hline \multirow[t]{2}{*}{ Variable } & \multicolumn{3}{|c|}{ Univariate analysis } \\
\hline & $\overline{H R}$ & $95 \% \mathrm{Cl}$ & $P$ value \\
\hline \multicolumn{4}{|l|}{ Sex } \\
\hline Male & 1 & & Ref \\
\hline Female & 0.777 & $0.534,1.130$ & $0.187^{\#}$ \\
\hline Age of disease onset (years) & 0.989 & $0.974,1.004$ & $0.141^{\#}$ \\
\hline Age of disease diagnosis (years) & 0.986 & $0.971,1.001$ & $0.059^{\#}$ \\
\hline \multicolumn{4}{|l|}{ History of allergic disease } \\
\hline No & 1 & & Ref \\
\hline Yes & 1.133 & $0.754,1.701$ & 0.548 \\
\hline Number of involved organs, $n$ & 1.050 & $0.965,1.143$ & 0.258 \\
\hline C3 (g/L) & 1.489 & $0.668,3.319$ & 0.330 \\
\hline C4 (g/L) & 0.218 & $0.023,2.089$ & $0.187^{\#}$ \\
\hline $\operatorname{ESR}(\mathrm{mm} / \mathrm{h})$ & 1.009 & $1.001,1.017$ & $0.035^{\#}$ \\
\hline CRP (mg/L) & 1.015 & $0.995,1.034$ & $0.140^{\#}$ \\
\hline Serum lgG4 (mg/dl) & 1.000 & $0.999,1.001$ & $0.147^{\#}$ \\
\hline Serum lgE (IU/ml) & 1.000 & $0.999,1.001$ & 0.772 \\
\hline \multicolumn{4}{|l|}{ Eosinophilia } \\
\hline No & 1 & & Ref \\
\hline Yes & 1.031 & $0.663,1.604$ & 0.892 \\
\hline \multicolumn{4}{|l|}{ Treatment } \\
\hline GC only & 1 & & Ref \\
\hline$G C+I S$ & 0.332 & $0.224,0.491$ & $<0.001^{\#}$ \\
\hline
\end{tabular}

$H R$ hazard ratio, $\mathrm{Cl}$ confidence interval, Ref reference, GC glucocorticoid, IS immunosuppressant. ${ }^{\#} P<0.2$
ROD patients were divided into initial glucocorticoid monotherapy group and initial glucocorticoid plus immunosuppressant therapy group. Oral glucocorticoids were given at a dose of $0.6-0.8 \mathrm{mg} / \mathrm{kg} /$ day in both groups and then tapered by $10 \mathrm{mg}$ every $2-4$ weeks. Immunosuppressants were selected according to the severity of the disease, patient's tolerance, and economic affordability, including cyclophosphamide, mycophenolate mofetil, methotrexate, leflunomide, azathioprine, iguratimod, and rituximab. The median duration of immunosuppressants was 21 (8-36) months. Respectively, the median relapse-free survival times for the initial glucocorticoid monotherapy group and the initial glucocorticoid plus immunosuppressant group were 17 months and 43 months, and the latter group had significantly longer relapse-free survival time. In addition, the log-rank test revealed that there was significant difference between the two relapse-free survival curves, the initial glucocorticoid plus immunosuppressant group had an obviously higher relapse-free survival rate than the initial glucocorticoid monotherapy group $(P<0.001)$.

\section{Discussion}

IgG4-RD is an immune-mediated systemic fibroinflammatory disease featuring tumefactive lesions, which can affect almost every organ system in the body. IgG4-ROD refers to IgG4-RD with ophthalmic manifestations and is one of the phenotypes of IgG4-RD. There are currently few studies focusing on IgG4-ROD, and no research has explored the differences in the clinical features between IgG4-ROD and nIgG4-ROD. Thus, it is difficult to establish the best treatment strategy for IgG4-ROD. Therefore, we conducted a large retrospective cohort study to compare demographic characteristics, clinical presentations, and laboratory parameters between IgG4ROD patients and nIgG4-ROD patients. In addition, the risk factors for IgG4-ROD relapse were also identified.

Currently, there is no exact data on the epidemiology of IgG4-ROD due to the lack of well recognition and wide understanding of IgG4-ROD. Several previous studies conducted in Japanese and US populations have reported that the frequency of IgG4-ROD ranges 4 to $34 \%$ of IgG4-RD patients [21-24]. While the frequency of IgG4-ROD in our cohort accounted for $58.8 \%$ of IgG4-RD patients. Maybe racial variations, sample size, or admission unit contribute to the difference of the frequency of IgG4-ROD in IgG4-RD.

Our research showed that IgG4-ROD patients had almost equal sex ratio, younger age of disease onset and diagnosis, and higher IgG4-RD RI. In addition, the initial lesion sites in majority of IgG4-ROD patients were mainly located in the ocular (41.6\%) and salivary gland (34.9\%), which were directly exposed to the external environment and lack barrier protection. The damage of 
a

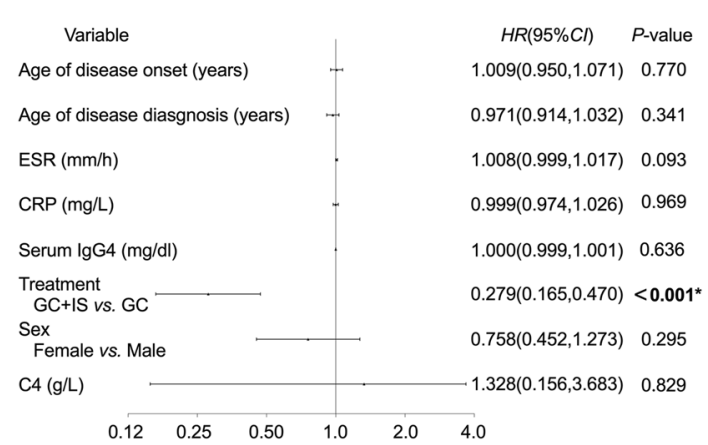

b

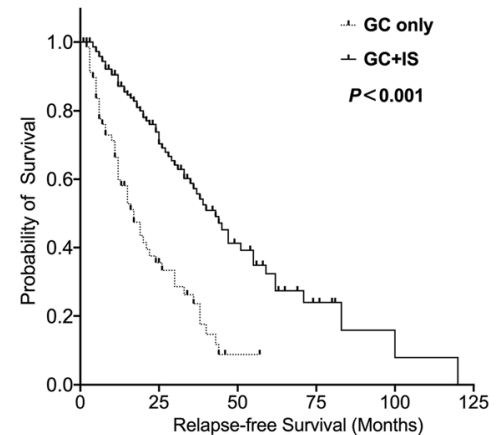

Fig. 3 Forest plot of multivariate Cox regression analysis and Kaplan-Meier curve for lgG4-ROD relapse. a Forest plot of multivariate Cox regression analysis for lgG4-ROD relapse. The initial glucocorticoid plus immunosuppressant was a protective factor for lgG4-ROD relapse $(P<$ 0.001). b Kaplan-Meier curve of relapse-free survival of IgG4-ROD patients based on the initial treatment options. IgG4-ROD patients treated with initial GC only vs IgG4-ROD patients treated with initial GC + IS ( $P<0.001)$. HR hazard ratio, Cl confidence interval, GC glucocorticoid, IS immunosuppressant. ${ }^{*} P<0.05$

tissue and immune tolerance to autoantigen in genetically susceptible individuals after exposure to certain environmental factors (allergens, bacteria, etc.) is considered to be a plausible mode of pathogenesis of IgG4-RD [2]. The reason why IgG4-ROD patients develop symptoms and get diagnosis at an earlier age, and have almost equal sex ratio may be that barrier-deficient organs can be exposed to the environmental triggers more easily, earlier, and with equal chance for both sexes.

IgG4-RD RI as a tool to evaluate the disease activity of IgG4-RD was used in this study. The higher IgG4-RD RI in IgG4-ROD group suggested that IgG4-ROD may have higher disease activity and may require more aggressive treatment. PET/CT is also considered to facilitate the management of IgG4-ROD, due to its good performance in detecting organ involvement, differentiating active and inactive lesions, and monitoring treatment response and relapse during IgG4-RD diagnosis and follow-up $[25,26]$; however, it is not covered by medical insurance in China-most of the patients did PET/CT to exclude tumor in our cohort.

With regard to the affected ocular anatomic structure in IgG4-ROD, we found that the lacrimal gland and extraocular muscle were most frequently involved, while other anatomic structures were rarely involved, which is highly in line with previous researches $[16,17]$. Similarly, eyelid swelling and xerophthalmia caused by lacrimal gland involvement were the most frequent manifestations in IgG4-ROD. More than $90 \%$ ( $\geq 2$ organs involved) of IgG4-ROD patients had extraocular manifestations, and the most common sites of extraocular involvement were the salivary gland and lymph node, which is similar with the previous study [27]. In addition, our study showed that IgG4-ROD patients had more organs involvement. Therefore, it is recommended that systemic examination and imaging evaluation should be carried out in IgG4-ROD patients to identify other organ involvement as early as possible and start systematic treatment.

In terms of laboratory parameters, IgG4-ROD patients had lower levels of ESR and CRP, which may reflect the absence of a higher activity of acute inflammation in IgG4-ROD. As we all know, elevated serum IgG4 concentration is one of the hallmark features of IgG4-RD. Previous studies have confirmed that serum IgG4 tends to be higher in IgG4-RD patients with the lacrimal or salivary gland $[21,28]$ and multiple organ involvement $[14,29]$. We also found that higher serum IgG4 levels and the IgG4/IgG ratio were found in IgG4-ROD patients in our study. Moreover, IgG4-ROD patients had higher serum IgE levels, higher frequencies of elevated IgE, and allergic disease.

It is currently believed that abnormal immune response against infection, allergen, or tissue injury may be the initiating factors in the pathogenesis of IgG4$\mathrm{RD}$, and then triggers can activate $\mathrm{T}$ follicular helper (Tfh) and $\mathrm{T}$ follicular regulatory (Tfr) cell immune response, and the subsequent production of IL-4/IL10 cytokines can promote the production and class switch of IgG4 and IgE in plasmablasts, eosinophil recruitment, and fibroblast activation [2, 30, 31] Finally, it leads to the onset of IgG4-RD. Recent studies have identified that CD4+ cytotoxic T lymphocytes (CTLs) may also play a central role in this pathophysiological process [32, 33]. It is well known that in some autoimmune diseases such as systemic lupus erythematosus, the emergence of certain autoantibodies is often associated with the heterogeneity in organ involvement and clinical manifestations [34]. IgG4-RD is 
thought to have similar profiles with the identification of diverse autoantigens that may serve as potential triggers [35-38]. Therefore, we suppose that differences in local allergen or autoantigen exposure may result in the different clinical and serological features of the two IgG4-RD phenotypes in this study. However, the exact mechanism remains unclear and needs further study.

The phenotypic differences of IgG4-RD have been more clearly understood after IgG4-RD was classified into four different groups: pancreato-biliary (group 1), retroperitoneum/aortitis (group 2), head and neck limited (group 3), and Mikulicz/systemic (group 4) [39]. When groups 3 and 4 are analyzed together, they can largely represent IgG4-ROD in this study. Compared with the results of IgG4-ROD in our study, group 3 plus 4 in the study with $42.2 \%$ of Asians [39] also has more organ involvement, ocular, salivary gland, paranasal sinus, lung, and lymph node involvement and higher serum IgG4 levels, but lower male to female sex ratio and retroperitoneal and biliary system lesions. However, another study with $95 \%$ of Caucasians seems to have higher levels of IgG4 and IgE in group 1 [40]. Therefore, possible genetic or ethnic factors also contribute to the clinical and serological differences between IgG4-ROD and nIgG4-ROD.

There are currently no clinical treatment guidelines to instruct the diagnosis and treatment of IgG4-ROD. The use of systemic glucocorticoids as first-line treatment for majority of IgG4-ROD patients usually results in the improvement of symptoms and decrease of serum IgG4 levels; however, the relapse is not uncommon during glucocorticoid tapering or withdrawal $[17,18]$. In this study, we found that initial glucocorticoid plus immunosuppressant was a protective factor for IgG4-ROD relapse. IgG4-ROD patients initially treated with glucocorticoid plus immunosuppressant had longer relapse-free survival time compared with the initial glucocorticoid monotherapy group. In our previous study, although there was the same trend as this one, we did not find a statistically significant correlation between the initial glucocorticoids plus immunosuppressants and the relapse of IgG4-RD [41]. The reason may be that the population and variables included in the two studies are different. Therefore, large cohort, prospective randomized controlled trials are still needed to evaluate the value of glucocorticoid plus immunosuppressants in the treatment of IgG4-ROD in future.

Our study has some limitations. First, our study suffers from the limitation of its retrospective design, nonrandomized controlled investigation, and treatment protocol. Second, this study was conducted in a single rheumatology center, thus possibly leading to a selection bias that cases with multiple organ involvements were reported.

\section{Conclusions}

In conclusion, our study demonstrated and confirmed on the largest cohort of IgG4-ROD patients that IgG4ROD showed younger age of disease onset and diagnosis, and almost equal sex ratio. IgG4-ROD group had higher AECC scores and IgG4-RD RI. Allergic diseases and multiorgan involvement were more frequent in IgG4-ROD group. Moreover, IgG4-ROD patients had higher serum IgG4 levels, IgG4/IgG ratio, and serum IgE levels. The salivary gland, paranasal sinus, lung, and lymph node involvement were more frequent in IgG4ROD patients. In addition, initial glucocorticoid plus immunosuppressant was a protective factor, which could prolong the relapse-free survival time of IgG4-ROD patients. These findings may have important implications for the understanding and management of IgG4-ROD.

\section{Abbreviations}

AECC: American College of Rheumatology/European League Against Rheumatism Classification Criteria; ANA: Antinuclear antibody; C3: Complement 3; C4: Complement 4; Cl: Confidence interval; CRP: Creactive protein; CT: Computed tomography; CTLs: Cytotoxic T lymphocytes; ESR: Erythrocyte sedimentation rate; GC: Glucocorticoid; HR: Hazard ratio; IgE: Immunoglobulin E; IgG: Immunoglobulin G; IgG4: Immunoglobulin G4; IgG4-RD: IgG4-related disease; IgG4-RD RI: IgG4-RD responder index; IgG4ROD: IgG4-related ophthalmic disease; IQR: Interquartile range;

IS: Immunosuppressant; MRI: Magnetic resonance imaging; nlgG4-ROD: NonIgG4-related ophthalmic disease; PET/CT: Positron emission tomography/ computed tomography; Ref: Reference; RF: Rheumatoid factor; SD: Standard deviation; Tfh: T follicular helper; Tfr: T follicular regulatory

\section{Acknowledgements}

Not applicable.

\section{Authors' contributions}

$Z Z, Y Y L$, and YS conceived and designed research; ZZ, ZQW, QZZ, ZFW, and JMX collected data; ZZ and DPM analyzed data, interpreted the results, and wrote the manuscript; LMR, YYL, and YS revised the manuscript. The authors read and approved the final manuscript.

\section{Funding}

This study was supported by grants from the Beijing Science and Technology Planning Project [Z191100006619111].

\section{Availability of data and materials}

The datasets used and/or analyzed during the current study are available from the corresponding author on reasonable request.

\section{Declarations}

Ethics approval and consent to participate

The Medical Ethics Committee of Peking University People's Hospital approved this study.

Consent for publication

Not applicable.

Competing interests

All authors have no conflicts of interest to disclose. 


\section{Author details}

'Department of Rheumatology and Immunology, Peking University People's Hospital, 11 Xizhimen South Street, Beijing 100044, China. ${ }^{2}$ Beijing Tongren Eye Center, Beijing Ophthalmology \& Visual Science Key Lab, Beijing Tongren Hospital, Capital Medical University, Beijing 100730, China.

Received: 17 October 2020 Accepted: 22 March 2021

Published online: 31 March 2021

\section{References}

1. Kamisawa T, Funata N, Hayashi Y, Eishi Y, Koike M, Tsuruta K, Okamoto A Egawa N, Nakajima H. A new clinicopathological entity of IgG4-related autoimmune disease. J Gastroenterol. 2003;38(10):982-4. https://doi.org/10.1 007/s00535-003-1175-y

2. Kamisawa T, Zen Y, Pillai S, Stone JH. IgG4-related disease. Lancet. 2015; 385(9976):1460-71. https://doi.org/10.1016/S0140-6736(14)60720-0.

3. Carruthers MN, Khosroshahi A, Augustin T, Deshpande V, Stone JH. The diagnostic utility of serum IgG4 concentrations in IgG4-related disease. Ann Rheum Dis. 2015;74(1):14-8. https://doi.org/10.1136/annrheumdis-2 013-204907.

4. Stone JH, Zen Y, Deshpande V. IgG4-related disease. N Engl J Med. 2012; 366(6):539-51. https://doi.org/10.1056/NEJMra1104650.

5. Liu Y, Xue M, Wang Z, Zeng Q, Ren L, Zhang Y, Zhang S, Wang Y, Shen D, Xia C, Yu G, Li ZG. Salivary gland involvement disparities in clinical characteristics of IgG4-related disease: a retrospective study of 428 patients. Rheumatology. 2020;59(3):634-40. https://doi.org/10.1093/rheumatology/ kez280.

6. Ebbo M, Daniel L, Pavic M, Sève P, Hamidou M, Andres E, Burtey S, Chiche L, Serratrice J, Longy-Boursier M, Ruivard M, Haroche J, Godeau B, Beucher AB, Berthelot JM, Papo T, Pennaforte JL, Benyamine A, Jourde N, Landron C, Roblot P, Moranne O, Silvain C, Granel B, Bernard F, Veit V, Mazodier K, Bernit E, Rousset H, Boucraut J, Boffa JJ, Weiller PJ, Kaplanski G, Aucouturier $P$, Harlé JR, Schleinitz N. IgG4-related systemic disease: features and treatment response in a French cohort: results of a multicenter registry. Medicine. 2012;91(1):49-56. https://doi.org/10.1097/MD.0b013e3182433d77.

7. Hedgire SS, McDermott S, Borczuk D, Elmi A, Saini S, Harisinghani MG. The spectrum of lgG4-related disease in the abdomen and pelvis. AJR Am J Roentgenol. 2013;201(1):14-22. https://doi.org/10.2214/AJR.12.9995.

8. Ozawa M, Fujinaga Y, Asano J, Nakamura A, Watanabe T, Ito T, Muraki T, Hamano H, Kawa S. Clinical features of IgG4-related periaortitis/periarteritis based on the analysis of 179 patients with lgG4-related disease: a casecontrol study. Arthritis Res Ther. 2017;19(1):223. https://doi.org/10.1186/s13 075-017-1432-8.

9. Yamada K, Yamamoto M, Saeki T, Mizushima I, Matsui S, Fujisawa Y, Hara S, Takahashi H, Nomura H, Kawa S, Kawano M. New clues to the nature of immunoglobulin G4-related disease: a retrospective Japanese multicenter study of baseline clinical features of 334 cases. Arthritis Res Ther. 2017;19(1): 262. https://doi.org/10.1186/s13075-017-1467-x.

10. Cheuk W, Yuen HK, Chan AC, Shih LY, Kuo TT, Ma MW, Lo YF, Chan WK, Chan JK. Ocular adnexal lymphoma associated with lgG4+ chronic sclerosing dacryoadenitis: a previously undescribed complication of IgG4related sclerosing disease. Am J Surg Pathol. 2008;32(8):1159-67. https://doi. org/10.1097/PAS.0b013e31816148ad.

11. Sato Y, Ohshima K, Ichimura K, Sato M, Yamadori I, Tanaka T, Takata K, Morito T, Kondo E, Yoshino T. Ocular adnexal IgG4-related disease has uniform clinicopathology. Pathol Int. 2008;58(8):465-70. https://doi.org/1 0.1111/j.1440-1827.2008.02257.x

12. Takahira M, Ozawa Y, Kawano M, Zen Y, Hamaoka S, Yamada K, Sugiyama K. Clinical aspects of IgG4-related orbital inflammation in a case series of ocular adnexal lymphoproliferative disorders. Int J Rheumatol. 2012;2012: 635473.

13. Maehara T, Moriyama M, Nakashima H, Miyake K, Hayashida JN, Tanaka A, Shinozaki S, Kubo Y, Nakamura S. Interleukin-21 contributes to germinal centre formation and immunoglobulin G4 production in IgG4-related dacryoadenitis and sialoadenitis, so-called Mikulicz's disease. Ann Rheum Dis. 2012;71(12):2011-9. https://doi.org/10.1136/annrheumdis-2012-201477.

14. Matsui S, Taki H, Shinoda K, Suzuki K, Hayashi R, Tobe K, Tokimitsu Y, Ishida M, Fushiki H, Seto H, Fukuoka J, Ishizawa S. Respiratory involvement in IgG4related Mikulicz's disease. Mod Rheumatol. 2012;22(1):31-9. https://doi.org/1 0.3109/s10165-011-0504-x.
15. Umehara H, Okazaki K, Masaki Y, Kawano M, Yamamoto M, Saeki T, Matsui S, Yoshino T, Nakamura S, Kawa S, Hamano H, Kamisawa T, Shimosegawa T, Shimatsu A, Nakamura S, Ito T, Notohara K, Sumida T, Tanaka Y, Mimori T, Chiba T, Mishima M, Hibi T, Tsubouchi H, Inui K, Ohara H. Comprehensive diagnostic criteria for lgG4-related disease (IgG4-RD), 2011. Mod Rheumatol. 2012;22(1):21-30. https://doi.org/10.3109/s10165-011-0571-z.

16. Ebbo M, Patient M, Grados A, Groh M, Desblaches J, Hachulla E, Saadoun D, Audia S, Rigolet A, Terrier B, Perlat A, Guillaud C, Renou F, Bernit E,

Costedoat-Chalumeau N, Harlé JR, Schleinitz N. Ophthalmic manifestations in IgG4-related disease: clinical presentation and response to treatment in a French case-series. Medicine. 2017;96(10):e6205. https://doi.org/10.1097/MD. 0000000000006205

17. Park J, Lee MJ, Kim N, Kim JE, Park SW, Choung HK, Khwarg SI. Risk factors for extraophthalmic involvement and treatment outcomes in patients with lgG4-related ophthalmic disease. Br J Ophthalmol. 2018;102(6):736-41. https://doi.org/10.1136/bjophthalmol-2017-310584.

18. Derzko-Dzulynsky L. IgG4-related disease in the eye and ocular adnexa. Curr Opin Ophthalmol. 2017;28(6):617-22. https://doi.org/10.1097/ICU. 0000000000000427

19. Wallace ZS, Khosroshahi A, Carruthers MD, Perugino CA, Choi H, Campochiaro C, Culver EL, Cortazar F, Della-Torre E, Ebbo M, et al. An international multispecialty validation study of the lgG4-related disease responder index. Arthritis Care Res. 2018;70(11):1671-8. https://doi.org/10.1 $002 /$ acr.23543

20. Wallace ZS, Naden RP, Chari S, Choi HK, Della-Torre E, Dicaire JF, Hart PA, Inoue D, Kawano M, Khosroshahi A, Lanzillotta M, Okazaki K, Perugino CA, Sharma A, Saeki T, Schleinitz N, Takahashi N, Umehara H, Zen Y, Stone JH, Members of the ACR/EULAR IgG4-RD Classification Criteria Working Group. The 2019 American College of Rheumatology/European league against rheumatism classification criteria for lgG4-related disease. Ann Rheum Dis. 2020;79(1):77-87. https://doi.org/10.1136/annrheumdis-2019-216561.

21. Hamano H, Arakura N, Muraki T, Ozaki Y, Kiyosawa K, Kawa S. Prevalence and distribution of extrapancreatic lesions complicating autoimmune pancreatitis. J Gastroenterol. 2006;41(12):1197-205. https://doi.org/10.1007/ s00535-006-1908-9.

22. Fujinaga $\mathrm{Y}$, Kadoya M, Kawa S, Hamano H, Ueda K, Momose M, Kawakami S, Yamazaki S, Hatta T, Sugiyama Y. Characteristic findings in images of extrapancreatic lesions associated with autoimmune pancreatitis. Eur J Radiol. 2010;76(2):228-38. https://doi.org/10.1016/j.ejrad.2009.06.010.

23. Takuma K, Kamisawa T, Anjiki H, Egawa N, Igarashi Y. Metachronous extrapancreatic lesions in autoimmune pancreatitis. Intern Med. 2010;49(6): 529-33. https://doi.org/10.2169/internalmedicine.49.3038

24. Wallace ZS, Deshpande V, Stone JH. Ophthalmic manifestations of IgG4-related disease: single-center experience and literature review. Semin Arthritis Rheum. 2014:43(6):806-17. https:/doi.org/10.1016/j.semarthrit.2013.11.008.

25. Ebbo M, Grados A, Guedj E, Gobert D, Colavolpe C, Zaidan M, Masseau A, Bernard F, Berthelot JM, Morel N, Lifermann F, Palat S, Haroche J, Mariette X, Godeau B, Bernit E, Costedoat-Chalumeau N, Papo T, Hamidou M, Harlé JR, Schleinitz N. Usefulness of 2-[18F]-fluoro-2-deoxy-D-glucose-positron emission tomography/computed tomography for staging and evaluation of treatment response in IgG4-related disease: a retrospective multicenter study. Arthritis Care Res. 2014;66(1):86-96. https://doi.org/10.1002/acr.22058.

26. Berti A, Della-Torre E, Gallivanone F, Canevari C, Milani R, Lanzillotta M, Campochiaro C, Ramirez GA, Bozzalla Cassione E, Bozzolo E, Pedica F, Castiglioni I, Arcidiacono PG, Balzano G, Falconi M, Gianolli L, Dagna L. Quantitative measurement of 18F-FDG PET/CT uptake reflects the expansion of circulating plasmablasts in lgG4-related disease. Rheumatology. 2017; 56(12):2084-92. https://doi.org/10.1093/rheumatology/kex234.

27. Wu A, Andrew NH, McNab AA, Selva D. Bilateral IgG4-related ophthalmic disease: a strong indication for systemic imaging. Br J Ophthalmol. 2016; 100(10):1409-11. https://doi.org/10.1136/bjophthalmol-2015-307437.

28. Zen Y, Nakanuma Y. lgG4-related disease: a cross-sectional study of 114 cases. Am J Surg Pathol. 2010;34(12):1812-9. https://doi.org/10.1097/PAS. Ob013e3181f7266b.

29. Igarashi H, Ito T, Oono T, Nakamura T, Fujimori N, Niina Y, Hijioka M, Uchida M, Lee $R$, Iwao $R$, et al. Relationship between pancreatic and/or extrapancreatic lesions and serum lgG and lgG4 levels in lgG4-related diseases. J Dig Dis. 2012; 13(5):274-9. https://doi.org/10.1111/j.1751-2980.2012.00583.x.

30. Mahajan VS, Mattoo H, Deshpande V, Pillai SS, Stone JH. IgG4-related disease. Annu Rev Pathol. 2014;9(1):315-47. https://doi.org/10.1146/annurevpathol-012513-104708. 
31. Perugino CA, Stone JH. IgG4-related disease: an update on pathophysiology and implications for clinical care. Nat Rev Rheumatol. 2020;16(12):702-14. https://doi.org/10.1038/s41584-020-0500-7.

32. Della-Torre $E$, Bozzalla-Cassione E, Sciorati C, Ruggiero E, Lanzillotta M, Bonfiglio S, Mattoo H, Perugino CA, Bozzolo E, Rovati L, Arcidiacono PG, Balzano G, Lazarevic D, Bonini C, Falconi M, Stone JH, Dagna L, Pillai S, Manfredi AA. A CD8a- subset of CD4+SLAMF7+ cytotoxic T cells is expanded in patients with lgG4-related disease and decreases following glucocorticoid treatment. Arthritis Rheumatol. 2018;70(7):1133-43. https:// doi.org/10.1002/art.40469.

33. Perugino CA, Kaneko N, Maehara T, Mattoo H, Kers J, Allard-Chamard H, Mahajan VS, Liu H, Della-Torre E, Murphy SJH, et al. CD4+ and CD8+ cytotoxic T lymphocytes may induce mesenchymal cell apoptosis in lgG4related disease. J Allergy Clin Immunol. 2020;50091-6749(20):30741-7.

34. Nagafuchi Y, Shoda H, Fujio K. Immune profiling and precision medicine in systemic lupus erythematosus. Cells. 2019;8(2):140. https://doi.org/10.3390/ cells8020140.

35. Du H, Shi L, Chen P, Yang W, Xun Y, Yang C, Zhao L, Zhou Y, Chen G. Prohibitin is involved in patients with lgG4 related disease. Plos One. 2015; 10(5):e0125331. https://doi.org/10.1371/journal.pone.0125331.

36. Hubers LM, Vos $H$, Schuurman AR, Erken R, Oude Elferink RP, Burgering $B$, van de Graaf SFJ, Beuers U. Annexin A11 is targeted by lgG4 and lgG1 autoantibodies in IgG4-related disease. Gut. 2018;67(4):728-35. https://doi. org/10.1136/gutjinl-2017-314548.

37. Shiokawa M, Kodama Y, Sekiguchi K, Kuwada T, Tomono T, Kuriyama K, Yamazaki H, Morita T, Marui S, Sogabe Y, et al. Laminin 511 is a target antigen in autoimmune pancreatitis. Sci Transl Med. 2018;10(453):eaaq0997.

38. Perugino CA, AlSalem SB, Mattoo H, Della-Torre E, Mahajan V, Ganesh G, Allard-Chamard H, Wallace Z, Montesi SB, Kreuzer J, et al. Identification of galectin-3 as an autoantigen in patients with IgG4-related disease. J Allergy Clin Immunol. 2019;143(2):736-745.e736.

39. Wallace ZS, Zhang Y, Perugino CA, Naden R, Choi HK, Stone JH. ACR/EULAR IgG4-RD classification criteria committee: clinical phenotypes of IgG4-related disease: an analysis of two international cross-sectional cohorts. Ann Rheum Dis. 2019;78(3):406-12. https://doi.org/10.1136/annrheumdis-2018-214603.

40. Lanzillotta M, Campochiaro C, Mancuso G, Ramirez GA, Capurso G, Falconi M, Dagna L, Della-Torre E. Clinical phenotypes of IgG4-related disease reflect different prognostic outcomes. Rheumatology. 2020;59(9):2435-42. https:// doi.org/10.1093/rheumatology/keaa221.

41. Liu Y, Zeng Q, Zhu L, Gao J, Wang Z, Wang Z, Yang F, Wang K, Chen D, Xia C, Zhang S, Wang Y, Shen D, Yu G, Li ZG. Relapse predictors and serologically unstable condition of IgG4-related disease: a large Chinese cohort. Rheumatology. 2020;59(8):2115-23. https://doi.org/10.1093/rheuma tology/kez669.

\section{Publisher's Note}

Springer Nature remains neutral with regard to jurisdictional claims in published maps and institutional affiliations.

Ready to submit your research? Choose BMC and benefit from:

- fast, convenient online submission

- thorough peer review by experienced researchers in your field

- rapid publication on acceptance

- support for research data, including large and complex data types

- gold Open Access which fosters wider collaboration and increased citations

- maximum visibility for your research: over $100 \mathrm{M}$ website views per year

At $\mathrm{BMC}$, research is always in progress.

Learn more biomedcentral.com/submissions 